\title{
Evolution of monolayer terrace topography on (100) GaAs annealed under an arsine/hydrogen ambient
}

\author{
J. E. Epler, T. A. Jung, ${ }^{\text {a) }}$ and H. P. Schweizer \\ Paul Scherrer Institute Zurich, Badenerstrasse 569, CH-8048 Zurich, Switzerland
}

(Received 15 June 1992; accepted for publication 26 October 1992)

\begin{abstract}
The topographical evolution of the (100) GaAs surface annealed under an arsine/hydrogen ambient is studied by in situ orientation-resolved light scattering and ex situ atomic force microscopy (AFM). The light scattering system provides real-time monitoring of the magnitude and crystal orientation of topographical features of $0.3 \mu \mathrm{m}$ scale. The AFM images of the GaAs surface, quenched at various annealing temperatures, vividly depict the randomly oriented high density monolayer steps evolving into an atomically smooth terracelike structure.
\end{abstract}

An essential preparatory process of semiconductor epitaxial growth is an in situ thermal annealing that desorbs surface contaminants and smooths the surface topography. For the case of GaAs under arsenic impingement, the annealing process and growth initiation have been studied in a molecular beam epitaxy (MBE) system where optical scattering, ${ }^{1,2}$ electron beam, ${ }^{3,4}$ and $\mathrm{x}$-ray photoelectron ${ }^{5-7}$ techniques are applicable. Based on these studies, $\mathrm{Ga}_{2} \mathrm{O}_{3}$ is the most stable component of GaAs native oxide, desorbing near $640^{\circ} \mathrm{C}$. Concurrent with the conclusion of the $\mathrm{Ga}_{2} \mathrm{O}_{3}$ desorption, chemical, and topographical restructuring of the surface is observed ${ }^{1}$ with reflection high energy electron diffraction and diffuse optical scattering, respectively.

In this letter, we report the application of in situ diffuse optical scattering ${ }^{8}$ in metalorganic chemical vapor deposition (MOCVD) system to study topographical restructuring from (100) GaAs under an arsine/hydrogen ambient. Uniquely, the in situ scattering signal is resolved with respect to crystal (substrate) orientation. Ex situ high quality atomic force microscope (AFM) images of the GaAs surface quenched at four stages of the microstructuring process are also shown. The results provide a greatly improved visualization of the topographical evolution from a high density of randomly oriented steps into a terracelike structure of monolayer steps. This topographical restructuring begins near $500^{\circ} \mathrm{C}$ and reaches a maximum rate of change near $640^{\circ} \mathrm{C}$. Our results suggest that the process of topographical reconstruction is relatively independent of the presence or absence of a naturally occurring surface oxide.

The MOCVD system used here is an Emcore GS-3200 with three optical ports added to the growth chamber. All three ports are inclined $73^{\circ}$ from the substrate normal and are located at $\Theta=0^{\circ}, 90^{\circ}$, and $180^{\circ}$ in the plane of the substrate. A $10 \mathrm{~mW}$ HeCd laser beam ( $442 \mathrm{~nm}$ ) enters the $0^{\circ}$ port, reflects off the center of the substrate, and exits the chamber through the opposing $\left(180^{\circ}\right)$ port. The diffusescattered light is collected through the $90^{\circ}$ optical port, passed through a narrow band interference filter and pinhole spatial filter, and detected with a photomultiplier. The

\footnotetext{
2) Present address: IBM T. J. Watson Research Center, Yorktown Heights, NY 10598.
}

signal is amplified ( $1 \mathrm{kHz}$ bandwidth) and digitized. The optical system selectively detects scattering from topographical Fourier components, $K$, of magnitude $19 \mu \mathrm{m}^{-1}$ corresponding to a feature size of $0.3 \mu \mathrm{m}$. Also, the symmetry of the optical system restricts the angle of $\mathbf{K}$ to $\Theta \approx 45^{\circ}$, thus creating a detection "window." Since in this growth system the substrate is rotated $(1000 \mathrm{rpm})$, we obtain in real time the orientation dependence of the topographical scattering.

The substrates are on-axis (100) $n^{+}$GaAs with "epitaxial ready" surface finish and, based upon the data below, are slightly misoriented $\left(0.032^{\circ}\right)$ toward the [001] direction. The substrates are loaded directly from the Fluoroware container (as received) into the loadlock of the MOCVD system. Alternatively, they are deoxidized in $10 \% \mathrm{HCl} / \mathrm{ethanol}^{5}$ and loaded with a minimum of air exposure. A $1 \%$ arsine in hydrogen annealing ambient (pressure $=90 \mathrm{mbar}$ ) is sufficient to prevent arsenic loss from the GaAs surface during the anneal. In Fig. 1 is shown the average scattering intensity (all orientations), the [001] scattering, and the sample temperature as a function of time during a typical pregrowth anneal of an asreceived wafer. [001] scattering refers to the momentary signal intensity when the substrate rotation brings $\mathbf{K}$ parallel to [001] into alignment with the detection window. For example, steps running along the [010] direction would have a large $\mathbf{K}$ parallel to [001]. The sample temperature (calibrated at the $\mathrm{Si}-\mathrm{Al}$ eutectic) is increased at $0.96{ }^{\circ} \mathrm{C}$ per s. A steady decrease in average scattering begins near $500{ }^{\circ} \mathrm{C}$ and continues to over $720^{\circ} \mathrm{C}$. Throughout this range the surface is becoming smoother as $\mathrm{Ga}$ mass transport $^{9}$ and evaporation ${ }^{10-14}$ reduce the density of monolayer steps. The steep drop in average scattering at $640^{\circ} \mathrm{C}$ is accompanied by the emergence of the dominant [001] component. As discussed below, at this characteristic restructuring temperature, $T_{n}$ the surface topography is evolving into a terracelike structure with monolayer steps of $0.5-\mu \mathrm{m}$ width, running along the [010] direction.

In Fig. 2, the orientation dependence of the in situ scattering of an as-received wafer is shown for several temperatures. For each curve the data from approximately 35 revolutions (2s) have been digitally averaged. A gradual evolution is observed from isotropic scattering, with a small enhancement near the [010] direction, towards a 


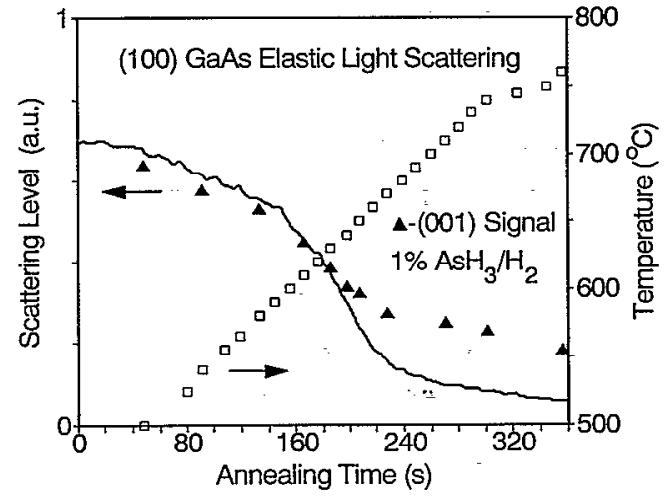

FIG. 1. The average elastic-light scattering intensity, all orientations (solid line) and [001] scattering (triangle), vs time. The annealing temperature (square) is also shown.

twofold symmetric [001] pattern as $T_{r}$ is exceeded. The [010] features are characteristic of wafers from this boule but are not understood. However, a twofold symmetric pattern always emerges near $T_{r}$ and is a reliable indicator of the final stage of the monolayer-terrace evolution.

To investigate the surface topography more directly, we use a commercially available AFM with force constants in the $0.03-0.35 \mathrm{~N} / \mathrm{m}$ range $\left(10^{-8} \mathrm{~N}\right.$ loading). A minimum required cantilever loading is optimum for imaging the surface steps. In Fig. 3 we show four AFM images after annealing an as-received wafer to: (a) 560, (b) 622, (c) 636 , and (d) $760^{\circ} \mathrm{C}$. The vertical scale (not shown) is different for each of the four images in order to optimize the contrast. However, in all cases the observed steps are approximately one monolayer $(0.28 \mathrm{~nm})$ in height. For this series the sample temperature is increased at a maximum rate $\left(2.5^{\circ} \mathrm{C} / \mathrm{s}\right)$ and then quickly cooled to quench the surface configuration. (The samples are generally exposed to atmosphere for several hours before the AFM measurements are made.) In Fig. 3(a) with $T<T_{r}$ the surface is composed of irregularly shaped (100) planar regions bounded by monolayer $(0.28 \mathrm{~nm})$ steps. There are a few double monolayer steps distributed throughout the scan area. In comparison an AFM image of an oxidestripped, unannealed sample (not shown) indicates an ill-

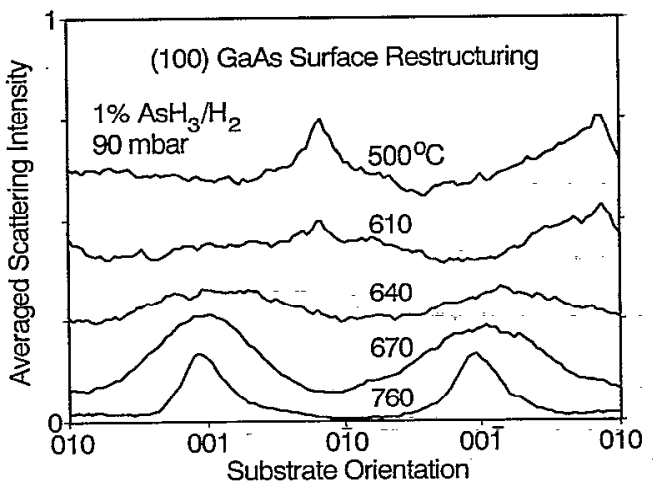

FIG. 2. The orientation dependence of the diffuse light scattering at several temperatures. For each curve 35 rotations are averaged to reduce the noise level.
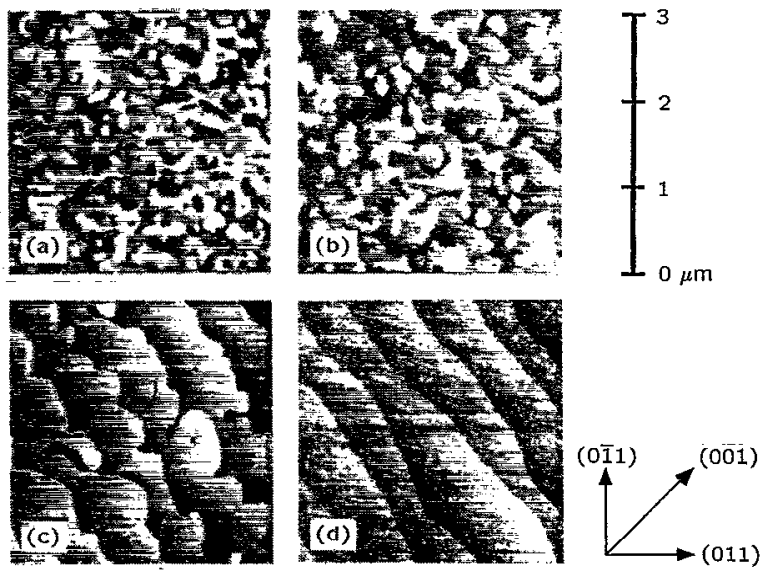

FIG. 3. AFM images of the surface topography after annealing in arsine/ hydrogen to (a) 560 , (b) 622 , and (c) 636 , and (d) $760^{\circ} \mathrm{C}$. The substrate orientation and lateral scale are indicated. (a) A high density of single and a low density of double monolayer steps are observed. (b) A roughedged terrace with numerous clusters and holes is observed. (c) At $T$, the average step density is rapidly decreasing. (d) The evolution of the terrace is complete.

defined surface of approximately $10 \mathrm{~nm} \mathrm{rms}$ roughness. Thus considerable surface restructuring on a $\mathrm{nm}$ scale has occurred at temperatures well below $T_{r}$

In Fig. 3(b), at $622^{\circ} \mathrm{C}$, the AFM image reveals a ragged terrace structure with monolayer steps running along the [010] direction. The numerous monolayer clusters and "holes" are of irregular shape, exhibiting no preferential crystal orientation. The average step density along the [001] direction has decreased from (a) 10 to (b) 5 per $\mu \mathrm{m}$. In (c), only $15^{\circ} \mathrm{C}$ higher in temperature at $T \approx T_{n}$ the edges of the terrace are much less ragged and the step density is only $2-3$ per $\mu \mathrm{m}$. More holes ( 3 per $10 \mu \mathrm{m}^{2}$ ) than clusters ( 1 per $10 \mu \mathrm{m}^{2}$ ) remain, as expected for a dissolution process. (Note that in contrast to a thick ozone $^{1}$ or chemical ${ }^{7}$ induced oxide, the desorption of the naturally occurring surface oxide does not result in pitting of the surface.) After the $760^{\circ} \mathrm{C}$ anneal, Fig. 3(d) shows the relatively regular monolayer terrace on the GaAs surface. The processes of $\mathrm{Ga}$ mass transport and evaporation have eliminated the clusters and holes and the in situ scattering is strongly anisotropic and stable.

We have obtained considerable data (not shown) on (100) GaAs substrates with chemically deoxidized surfaces and various misorientations. The results consistently indicate that $T_{r} \approx 640^{\circ} \mathrm{C}$ is the temperature at which the final stage of topographical restructuring quickly develops. In addition to the published work ${ }^{1,3}$ described in the first paragraph, MBE growth studies ${ }^{10-12}$ have concluded that the evaporation rate from a (100) GaAs surface becomes appreciable at $T_{r} \approx 640^{\circ} \mathrm{C}$ and is "limited by surface decomposition." And finally, investigations of GaAs evaporation under Knudsen ${ }^{13,14}$ and Langmuir ${ }^{14}$ conditions give $627(637)^{13}$ and $657^{\circ} \mathrm{C}$, respectively, as the congruent evaporation point of GaAs. In general, the available data support the contention that a key step in thermal decomposition of $\mathrm{GaAs}$ is activated near $640^{\circ} \mathrm{C}$. One possibility is that liberation of partially coordinated $\mathrm{Ga}$ (residing at rel- 
atively stable kink sites, for example) is occurring and is the rate limiting step for $\mathrm{Ga}$ evaporation and monolayer terrace formation. The observed desorption ${ }^{3,7}$ of $\mathrm{Ga}_{2} \mathrm{O}_{3}$ near $T_{r}$ may be a result more than a cause of topographical changes. However, further study is required to clarify the degree of independence of $\mathrm{Ga}$ mass transport, evaporation, and oxide desorption. Another issue for further consideration is the influence of arsine, or the monoatomic hydrogen produced by the dissociation of arsine, on these processes.

In conclusion, in situ angle-resolved diffuse optical scattering and AFM have been applied to the problem of GaAs oxide desorption and surface reconstruction under an arsine/hydrogen ambient. The AFM images graphically illustrate the evolution of the surface topography from a high density of random monolayer steps $\left(<540^{\circ} \mathrm{C}\right)$ to a highly ordered atomic terrace. The thermal liberation of $\mathrm{Ga}$ from relatively stable kink sites is suggested as the mechanism that enables the rapid restructuring at $640^{\circ} \mathrm{C}$.

We would like to acknowledge the many discussions on surface topographical mapping with Dr. E. F.
Steigmeier and the encouragement and general assistance of Dr. H. W. Lehmann.

'G. W. Smith, A. J. Pidduck, C. R. Whitehouse, J. L. Glasper, A. M. Keir, and C. Pickering, Appl. Phys. Lett. 59, 3282 (1991).

${ }^{2}$ D. E. Aspnes, W. E. Quinn, and S. Gregory, Appl. Phys. Lett. 56, 2569 (1990).

${ }^{3}$ A. J. SpringThorpe, S. J. Ingrey, B. Emmerstorfer, P. Mandeville, and W. T. Moore, Appl. Phys. Lett. 50, 77 (1987).

${ }^{4}$ F. Briones, D. Golmayo, L. Gonzalez, and J. L. De Miguel, Jpn. J. Appl. Phys. 24, L478 (1985).

${ }^{5}$ R. P. Vasquez, B. F. Lewis, and F. J. Grunthaner, J. Vac. Sci. Technol. B 1, 791 (1983).

'J. Massies and J. P. Contour, J. Appl. Phys. 58, 806 (1985).

${ }^{7}$ T. Van Buuren, M. K. Weilmeier, I. Athwal, K. M. Colbow, J. A. Mackenzie, T. Tiedje, P. C. Wong, and K. A. R. Mitchell, Appl. Phys. Lett. 59, 464 (1991).

${ }^{8}$ J. M. Olson and A. Kibbler, J. Cryst. Growth 77, 182 (1986).

${ }^{9}$ Z. L. Liau and H. J. Zeiger, J. Appl. Phys. 67, 2434 (1990).

${ }^{10}$ T. Kojima, N. J. Kawai, T. Nakagawa, K. Ohta, T. Sakamoto, and M. Kawashima, Appl. Phys. Lett. 47, 286 (1985).

${ }^{11}$ R. Heckingbottom, J. Vac. Sci. Technol. B 3, 572 (1985).

${ }^{12}$ J. M. Van Hove and P. I. Cohen, Appl. Phys. Lett. 47, 726 (1985).

${ }^{13}$ J. R. Arthur, J. Phys. Chem. Solids 28, 2257 (1967).

${ }^{14}$ C. T. Foxon, J. A. Harvey, and B. A. Joyce, J. Phys. Chem. Solids 34, 1683 (1973). 